\title{
Temperature Dependence of Quantum Dots- In-Well Infrared Photo Detectors (QDIPs) using Photoluminescence
}

\author{
Noor H Jabarullah
}

\begin{abstract}
A research of quantity dots-in-well infrared photo detectors (QDIPs) produces helpful outcomes for creating a twocolor QDIP. Quantum dot infrared photo detectors (QDIPs) have been shown to be a main technology in mid-and longwavelength infrared detection owing to their capacity for normal incidence operation and low dark current. This research explores infrared detectors based on intersubband transitions in a novel heterostructure of InAs / InO.15 Ga0.85 As / GaAs quantum dotsin-well (DWELL). The InAs quantum dots are also positioned in an InO.15 Ga0.85 in the DWELL framework, which in turn is well positioned with the In0.1Ga0.9As obstacle in GaAs quantum. Using fourier transform infrared spectroscopy, the optical characteristics of the sample were researched using photoluminescence and photocurrent. Spectrally adjustable reaction was noted at $6.2 \mu \mathrm{m}$ and $7.5 \mu \mathrm{m}$ with prejudice and lengthy wave IR reaction.
\end{abstract}

\section{INTRODUCTION}

High performance infrared (IR) photodetection is desirable for many applications involving thermal imaging, chemical analysis, night vision, remote sensing, space range, mine detection and fiber optic communications. Depending on the implementation, one may want either distinct requirements, either a wide, multicolor response from the detector, or a sharp single wavelength reaction. In addition to generating pictures with some color depth, multicolor IR detection has the capacity to determine the observed body temperature, so it is much more advantageous for a particular imaging scheme. Multi-color focal plane array (FPA) photodetectors are particularly helpful for medical and military imaging as well as environmental monitoring applications.

Quantum dots-in-well DWELL is component of a fresh technology of nano-photonic semiconductor devices, using stacked layers of quantum dots (QDs) as the active region in their constructions. DWELL photodetectors are a promising fresh advance that will hopefully overcome many of the shortcomings of current IR photodetection technologies. QDIPs use inter-subband absorption to detect small absorption, $3-5 \mu \mathrm{m} \quad \& \quad 8-14 \mu \mathrm{m}$ atmospheric' windows' medium and long wavelength light radiation.
Significant research has been carried out into the study of QDIPs, but little in the way of multi-color QDIP development, despite their desirability for a wide variety of applications. The objective of this project is to acquire the sample's optical properties through photoluminescence and photocurrent measurement while analyzing the benefits and disadvantages of the quantum-dots-in-well photodetector. This paper involves studying the mixture or hybrid of quantum-dot and quantum-well, known as dot-in-well (DWELL), and optimizing the benefits of the QDIP hypothesis by this hybrid system. The aim of this paper is to study the optical properties of the DWELL QDIP sample

using experimental techniques. The experimental work on this project focuses studying the optical properties by the means of photoluminescence and photocurrent.

\section{ENERGY BAND STRUCTURE}

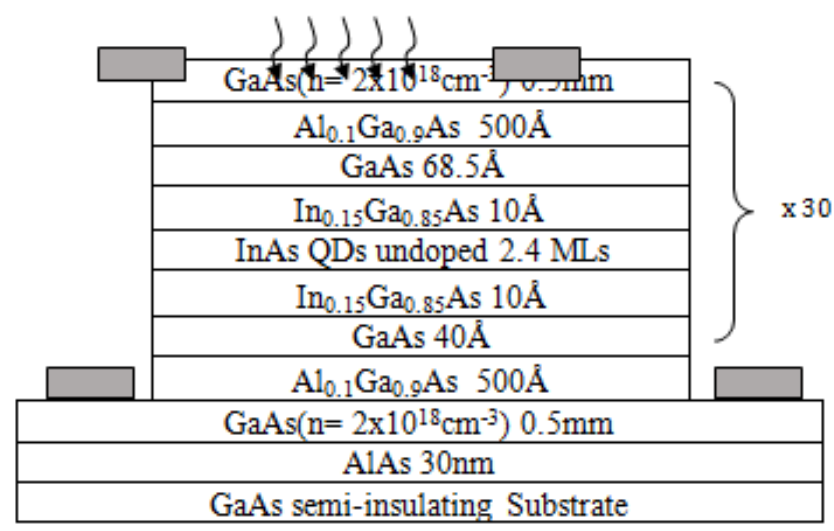

Fig. 1 Hetero structure of schematic of InAs/ $\mathrm{In}_{0.15} \mathrm{Ga}_{0.8} \mathrm{As} / \mathrm{GaAs}$ dots-in-well infrared photo detector 


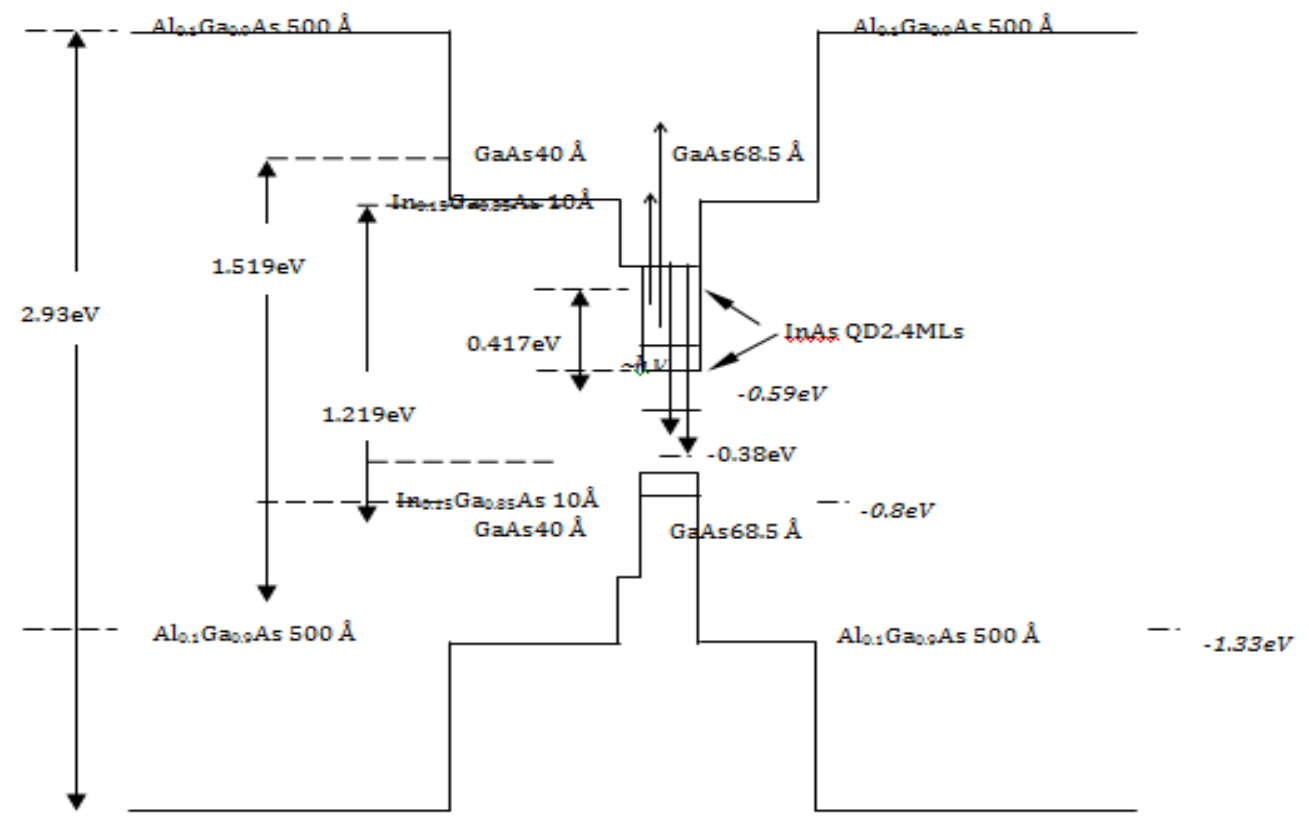

Fig. 2 The unstrained energy band structure

Figure 1 shows schematic of the DWELL structure and the energy band profile for the sample structure is illustrated in Figure 2. The valence band offset (VBO) of the
$\mathrm{Al}_{0.1} \mathrm{Ga}_{0.9} \mathrm{As}, \mathrm{GaAs}, \mathrm{In}_{0.15} \mathrm{Ga}_{0.8} \mathrm{As}$ and InAs is $-1.33 \mathrm{eV}$, $0.8 \mathrm{eV}, \quad-0.38 \mathrm{eV}$ and $-0.59 \mathrm{eV}$ respectively.

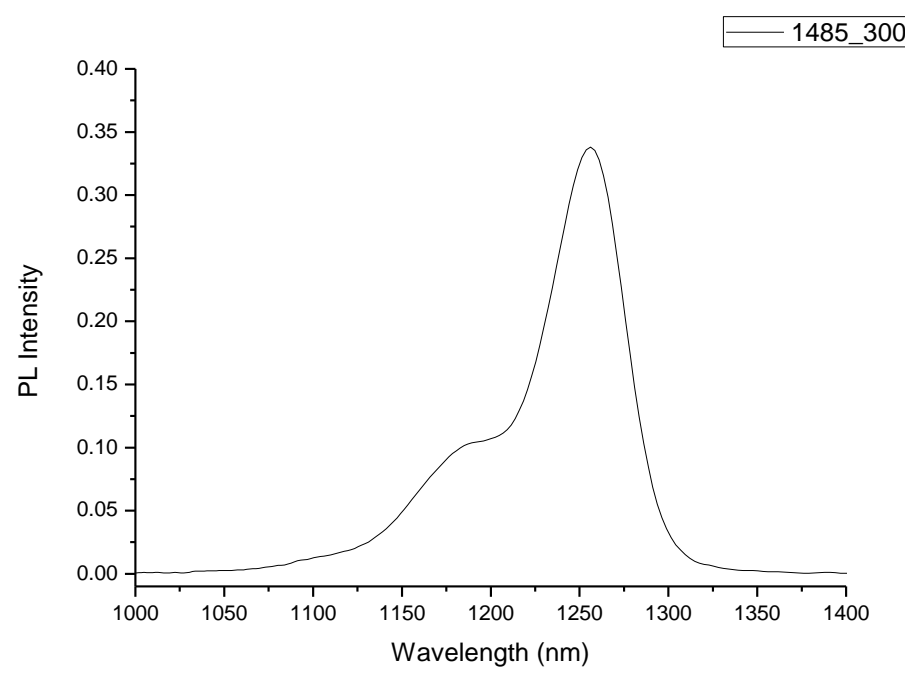

Fig. 3 Room temperature PL for the 30-period this InAs/ $\operatorname{In}_{0.15} \mathrm{Ga}_{0.8} \mathrm{As} / \mathrm{GaAs}$

The room temperature PL spectrum obtained from this InAs/ $\mathrm{In}_{0.15} \mathrm{Ga}_{0.8} \mathrm{As} / \mathrm{GaAs}$ dots-in-well infrared photodetector QDIP is shown in Figure 3.13. The PL wavelength of the InAs is $1255 \mathrm{~nm}$ showing that this device is crucial to achieve quantum efficiency without introducing dislocations.

Infrared photodetectors with an $\mathrm{InAs} / \mathrm{In}_{0.15} \mathrm{Ga}_{0.8} \mathrm{As} / \mathrm{GaAs}$ DWELL active region suffer from strain related issues. Due to high compressive strain, a greater number of active region layers cannot be grown without introducing dislocations. More layers will enhance the absorption, which in turn increases the quantum efficiency, $\eta$ of the photodetector. This increase in $\eta$ will increase the responsivity and detectivity of the detectors.

In order to determine the energy levels it is necessary to include the effects due to strain on the structure. The energy values of the QW's are affected by the strain in the layers (Osbourn, 1982). GaAs has a direct energy gap at the $\Gamma$ point of 1.519 and InAs has a direct energy gap of 0.417 at the $\Gamma$ point. Thus the value of $\mathrm{In}_{0.15} \mathrm{Ga}_{0.8} \mathrm{As}$ is less of $\mathrm{GaAs}$ and has been obtained from the ternary calculation depending on the alloy composition using a simple quadratic equation;

$E_{g}\left(A_{1-x} B_{x}\right)=(1-x) E_{g}(A)+x E_{g}(B)+x(1-x) C(3.1)$ where $\mathrm{C}$ is the bowing parameters that has been obtained from linear interpolation between the two A and B binaries (Vurgaftman \& Meyer, 2001).

Published By: 
The lattice mismatch between the substrate, the QWs and the other material in the sample is not too large, so when the mismatch is less than $7 \%$, it is assumed that no misfits are generated at the interfaces by compression or expansion of the planes (Osbourn, 1982) and (Vurgaftman \& Meyer, 2001). This means that the strain in the materials consist of uniform biaxial strain (hydrostatic) parallel to the interface and unaxial strain parallel to the growth direction. The hydrostatic component of compressive strain increases the bandgap and tensile strain reduces the bandgap. The strain effects not only on the band edges but also modifies the effective massed associated with the individual bands. The strained-induced of the bulk material responds to the shift of energies in QWs and barrier heights. The hydrostatic strain is obtained using below equation;

$$
\begin{gathered}
\varepsilon_{\|}=\varepsilon_{x x}=\varepsilon_{y y}=\frac{a_{s}-a_{e}}{a_{e}} \\
\varepsilon_{z z}=-2 \frac{C_{12}}{C_{11}}
\end{gathered}
$$

where $\varepsilon_{\mathrm{xx}}, \varepsilon_{\mathrm{yy}}$ and $\varepsilon_{\mathrm{zz}}$ are diagonal strain components of strain tensor is defined to be negative for compressive strain. $a_{s}$, is the lattice constant for the substrate and $a_{e}$, is the epilayer lattice constant. $\mathrm{C}_{12}$ and $\mathrm{C}_{11}$ is the elastic constants. The material parameters for the calculation of bandgap, VBO and strain are taken from Vurgaftman\& Meyer (2001).

Energy bandgap change in the conduction band, $\Delta \mathrm{E}_{\mathrm{c}}$ and valence band, $\Delta \mathrm{E}_{\mathrm{v}}$ is given by;

$$
\begin{aligned}
& \Delta E_{c}=a_{c}\left(\varepsilon_{x x}+\varepsilon_{y y}+\varepsilon_{z z}\right) \\
& \Delta E_{v}=a_{v}\left(\varepsilon_{x x}+\varepsilon_{y y}+\varepsilon_{z z}\right)
\end{aligned}
$$

From the Bir-Pikus strain interaction it is not sufficient to describe the full effect of the valence band strain. Shear deformation potential, $b$ is necessary to explain the shear deformation that split the valence band into heavy/light hole.

The + and - signs refer to heavy and light hole respectively.

In order to calculate the $\Delta E_{c}$ and $\Delta E_{v}$ for the ternary materials linear interpolation between the binary data obtain from Vurgaftman \& Meyer (2001) is used to determine the values if the parameters used.

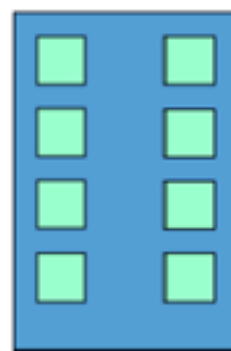

(a)

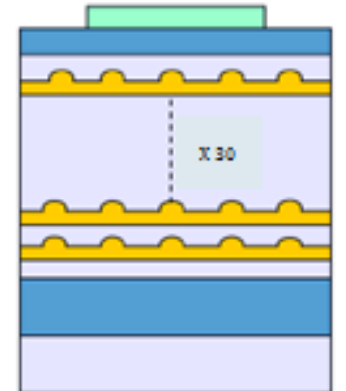

(b)

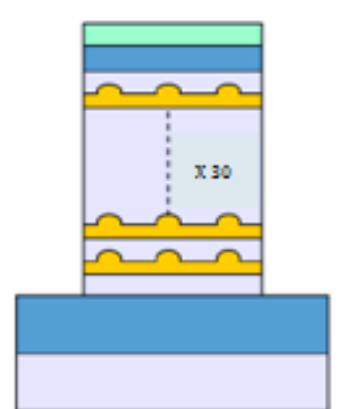

(c)

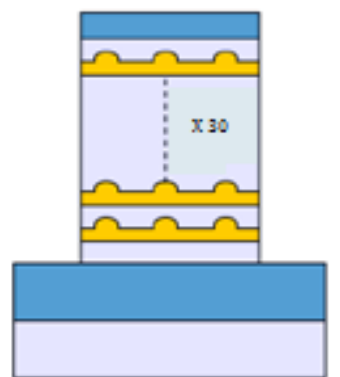

(d)

Fig. 4 The schematic of the mesa structure processes. (a) The top view of the patterned sample, with the photoresist marked in light blue. (b) The sample cross-section with the photoresist square on top. (c) Cross-section etching process. (d) A single mesa cross-section after photoresist been removed - reproduced from $\operatorname{Sze} \& \mathrm{Ng}(2007)$. 
The second phase was the formation of metal contacts and the final packaging. Another photolithography step was taken to identify regions for metal contacts on the QDIPs. The lithography operation outlined in the above steps was repeated using a distinct metal contact mask. The pattern for the contacts was to leave transparent circles of $200 \mu \mathrm{m}$ diameter on top of each mesa and long transparent lines on the bottom contact layer, with the remainder of the collection covered in photoresist. This will allow each sample QDIP to have an autonomous top contact and a shared bottom contact. After the second photolithography phase, metal contacts were created first by washing the sample in $\mathrm{HCl}$ (10 percent) for $40 \mathrm{~s}$ to remove natural oxidation. The technique of evaporation was placed for the n-type metal contacts using layers of $\mathrm{Ge}, \mathrm{Ni}$ and $\mathrm{Au}$. After that is the lift-off process by using acetone to remove the photoresist from the sample. The final step consists of annealing the contacts for 45 seconds using rapid thermal annealing under $\mathrm{N} 2$ flow at $400^{\circ} \mathrm{C}$. The unit was lastly packaged by mounting it on a 12-pin TO5 header, as shown in Figure 3.7, with $25 \mu \mathrm{m}$ thick gold cables attached to the metal contacts on all the QDIPs in the sample, connecting them to the gold buttons on the header. This stage is essential to characterize the sample using photocurrent measurement.

\section{Photoluminescence (PL) Measurement}

Photoluminescence (PL) is the spontaneous emission of light from the optical excitation of a material used to explore the sample's optical properties. PL studies can be used to characterize both inherent electronic transitions and impurity electronic transitions as well as semiconductor and insulator defects. PL is the typical method used to characterize III-IV semiconductors owing to the benefit of non-destructive, non-electrical contact requiring minimal effort for sample preparing. PL detects the optical shift from an excited electronic state to lower electronic state, generally ground state (Figure 3.15). Due to fast thermalization, only transitions from the reduced electronic states can usually be noted at low temperatures. Most of the light outcomes from the excited electron's energy difference returning to its ground state.

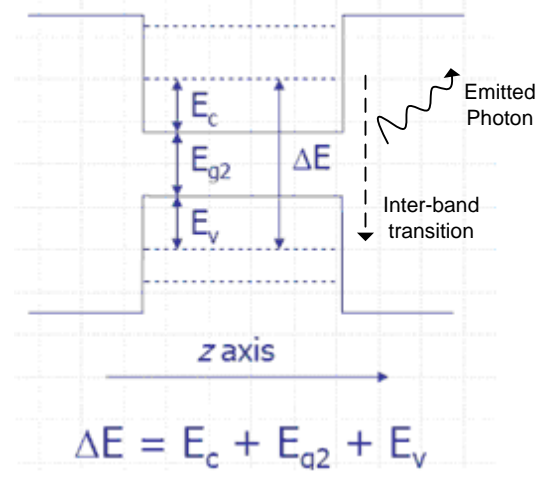

Fig. 5 Photoluminescence mechanism and relationship with electronic band structure
The PL spectra have narrow band which makes the analysis possible (Strading \& Klipstein, 1990). The position of PL peaks is related to the energy of each excited level and can be used as sensitive probe to find impurities and other defects in semiconductors. The PL intensity can give more information on the quality of interfaces and surfaces. PL spectrum and the dependencies on its intensity on the irradiation intensity and device temperature provide important information for device characterisation. PL spectra and the intensity can be used determine the energy gap or wavelength of maximum gain, understand the component of ternary/quaternary layers its impurity levels and allows for the understanding and investigations of the recombination mechanism investigations (Gfroerer, 2000)

The PL measurement were completed using a Fourier Transform Infrared (FTIR) spectroscopy, FTIR is an efficient and dynamic technique that provides qualitative and quantitative information which require a broadband range from Near-IR to Far-IR spectrum. FTIR spectrometer collects and examines all wavelengths simultaneously instead of viewing each one separately. This technique is known as Felgett or multiplex advantage. The interferometer will produce infrared spectra by collecting the interferogram of a sample that involves Fourier Transform mathematical manipulation that can involve phase correction and apodization.

\section{Photocurrent (PC) Measurement}

In contrast to other absorption-like spectroscopy, photocurrent (PC) is a comparatively simple, fast and easy to acquired technique, which reveals clear and unambiguous excitonic absorptions of self-assembled QDs. In the PC spectroscopy, the undergone processes of the photogenerated carriers are complementary to the PL measurements.

\section{RESULTS AND DISCUSSIONS}

\section{Temperature Dependence}




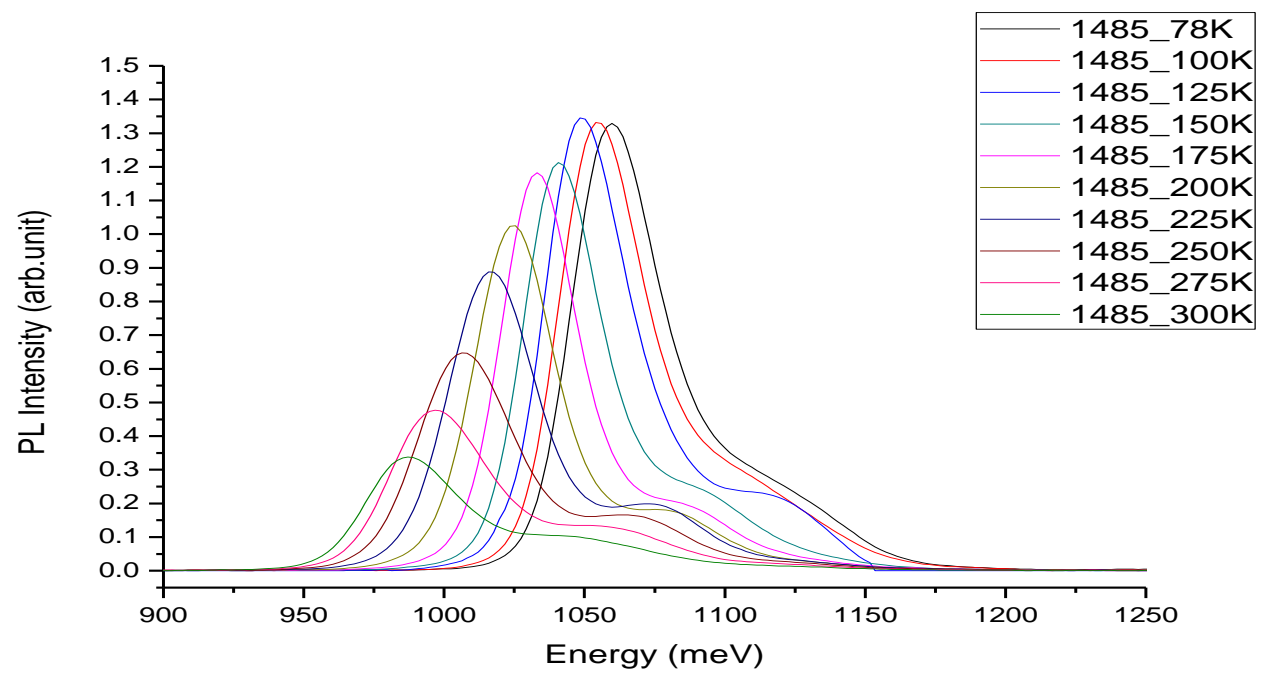

Fig. 6 PL spectra for sample VN1485 at temperatures from 78K to $300 \mathrm{~K}$

The spectra show a main peak, with a higher energy (ground state) lower intensity (excited state) shoulder. As temperature is reduced, the spectrum shifts towards the red which is to higher wavelengths. As to the variation of peak emission intensity with temperature, it appears that the peak intensity increases slightly as temperature is increased from $78 \mathrm{~K}$, peaking at $125 \mathrm{~K}$, before reducing substantially towards $300 \mathrm{~K}$.
For purposes of clarity, the spectra in Figure 4.1 were all normalized and plotted in Figure 4.2. It is clear that the spectral main peak red-shifted with reducing of temperature, where at $78 \mathrm{~K}$ the ground state peak is at $2.24 \mu \mathrm{m}$ and reducing to $1.9 \mu \mathrm{m}$ at $300 \mathrm{~K}$. The higher energy shoulder is visible in each spectrum as well.

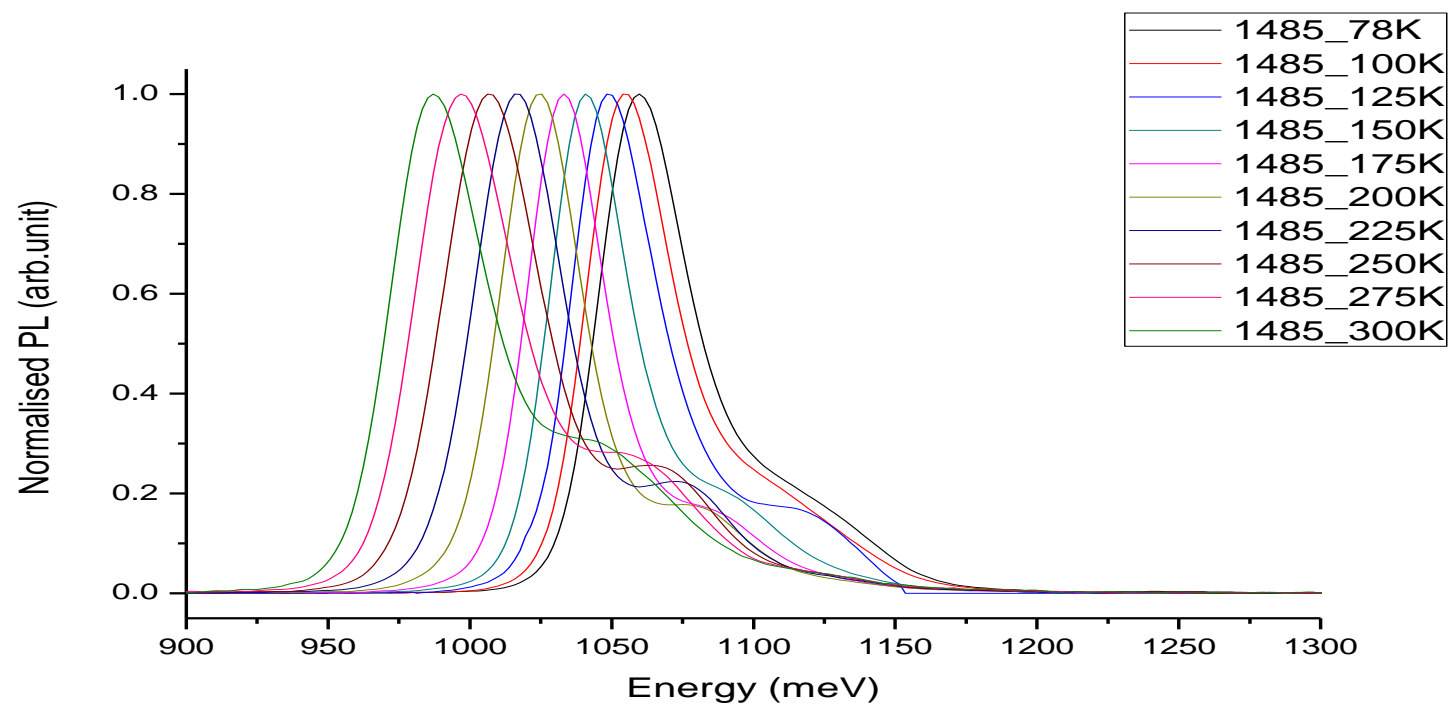

Fig. 7 Normalised PL spectra for sample VN1485 at temperatures from $78 \mathrm{~K}$ to $300 \mathrm{~K}$

To aid in the analysis, the data was fitted to two Gaussian curves (e.g. Figure 4.3 , at $225 \mathrm{~K}$ ), the sum of which would account for a main peak and shoulder. The curve fitting tool in OriginLab was applied to the spectral data, with fits made to using fit linear curve. The integrated intensity was then obtained by numerical integration of the Gaussian curves.

At $78 \mathrm{~K}$ the PL transition energy is found to be $1.14 \mathrm{eV}$ from the experimental result, and comparing to the modelling calculation provided by the optoelectronics research group it is assumed that the $\mathrm{Ga}$ content, $\mathrm{x}$ in the InAs dot is between $30 \%$. The modelling result is based on undoped material only therefore no comparison can be made for the doped sample.

\section{CONCLUSION}

This paper has presented the mid-wavelength infrared QDIP with 30-layers of DWELLs in the active region where special attention has been given to the optical properties of the intersubband photodetector namely the DWELL detector. The photoluminescence and photocurrent of the device has been investigated.

Published By: 
The results showed impressive results that are good control over the operating wavelength, higher operating temperature and adjustable spectral bias. The DWELL's photoresponse showed a reliance on the applied bias owing to the tilting of the edge of the conductive band and the shift in the probability of tunneling through the obstacles from the Quantum well excited states. By changing the bias it is possible to tune the wavelength window of the detector.

\section{REFERENCES}

1. A.Y.Cho. (1971). Film Deposition by Molecular Beam Techniques. Journal of Vaccum Science Technology (8), 31-38.

2. A.Y.Cho. (1979). Recent Developments in structures Molecular Beam Epitaxy (MBE). Journal of Science Technology , 16, 275-284.

3. Broekart, T. P., \&Fonstad, C. (1992). Novel Organic Acid Based Etchants for InGaAlAs/InPHeterostructure Devices with AlAs EtchStop Layers. Journal of Electrochemical Society , 139 (8).

4. Chang, L., L.Esaki, Howard, W., R.Ludeke, \&G.Schul. (1973). Structures Grown by Molecular Beam Epitaxy. Journal of Vacuum Science Technology (10), 655-662.

5. Colorado, U. o. (2010). IR Spectroscopy Tutorial. Retrieved August 25, 2010, from Spectroscopy: http://orgchem.colorado.edu/hndbksupport/irtutor/main.html

6. Gupta, R., Lai, K. T., Missous, M., \& Haywood, S. K. (2004) Subbandnonparabolicity estimated from multiple intersubband absorption in highly doped multiple quantum wells. Physical Review B , 69 (3), 033303.

7. Gupta, R., Wong, A. L., \& Haywood, S. K. (1999). $3 \mu \mathrm{m}$ intersubband Quantum Well Photodetector (QWIP). Tr. Journal of Physics , 23, 673679.

8. Haywood, S. K., Lai, K. T., \&Missous, M. (2006). Mid-infrared Semiconductor Optoelectronics. Berlin: Springer.

9. Krishna, S. (2005). Quantum dots-in-a-well infrared photodetectors. Journal of Physics D: Applied Physics, 38 (13), 2142-2150.

10. Levine, B. F. (1993). Quantum-well infrared photodetectots. Journal of Applied Physics, 74 (8), 1-81.

11. Ling, H. S., Wang, S. Y., Lee, C. P., \& Lo, M. C. (2008). High quantum effieciency dots-in-a-well quantum dot infrared photodetectors with $\mathrm{AlGaAs}$ confinement enhancing layer. Applied Physics Letters, 92 (19), 193506.

12. Liu, H. C. (2003). Quantum dot infrared photodetector. OptoepectronicsReview, 11, 1-5.

13. Martyniuk, P., Krishna, S., \&Rogalski, A. (2008). Assessment of quantum dot infrared photodetectors for high temperature operation. Journal of Applied Physics , 104 (3), 034314

14. Mashade, M. B., Ashry, M., \& Nasr, A. (2003). Therotical analysis of quantum dot infrared photodetectors. Semiconductor Science and Technology , 18 (9), 891-900.

15. Micaleff, J., \&Wiess, B. L. (1990). The refractive index of III-Iv semiconductor strained-layer superlattices. Optical and Quantum Electronics , 669-684.

16. Osbourn, G. (1982). Journal of Applied Physics. Strained layer superlattices from lattice mismatch materials , 53, 1586-1589.

17. Parker, G. (1994). Introductory Semiconductor Device Physics. Prentice Hall International (UK) Limited.

18. Philips, J. (2002). Evaluation of the fundamental properties of quantum dot infrared detectors. Journal of Applied Physics , 91 (7), 4590-4594.

19. Pryor, C., \& Pistol, M. (2005). Bande-edge diagrams for strained III-IV semiconductor quantum wells, wires and dots. Physical Review B , 72, 1098-1121.

20. Rhyzii, V. (1997). Characteristics of quantum well infrared photodetectors. Journal of Applied Physics , 81 (9), 6442-6448.

21. Rinaldi, F. (2002). Annual Report Optoelectronics Department. University of Ulm

22. Rogalski, A. (2009). Outlook on Quantum dot infrared photodetectors. Optical Memory and Neural Networks (information Optics) , 18 (3), 234-252.

23. Rogalski, A. (2002). Quantum Well Infrared Photoconductors In Infrared Detection Technology. International Journal of High Speed Electronics and Systems, 12 (3), 593-658.

24. Rogalski, A. (2003). Quantum well photodetectors in infrared detector technology. Journal of Applied Physics, 93 (8), 4355-4391.
25. Schneider, H., \& Liu, H. C. (2007). Quantum Well Infrared Photodetectors Physics and Applications. Heidelberg: Springer.

26. Shenoi, R. V., Attaluri, R. S., Siroya, A., Shao, J., Sharma, Y. D., Stinz, A., et al. (2008). Low Strain InAs/InGaAs/ quantum dots-in-a well infrared photodetector. Journal Vaccum Science Technology , 26 (3), 1136-1139.

27. Strading, R. A., \&Klipstein, P. C. (1990). Growth and characterisation of semiconductors. Bristol: J W Arrowsmith Ltd.

28. Sze, S. M., \& Ng, K. K. (2007). Physics Of Semiconductor Devices. John Wiley \& Sons.

29. Vurgaftman, I., \& Meyer, J. R. (2001). Band parameters for III-IV compound semiconductor and thier alloys. Applied physics review , 89 (11), 5818-5875.

30. Wilson, J., \& Hawkes, J. (1998). Optoelectronics an Introduction. Prentice Hall. 